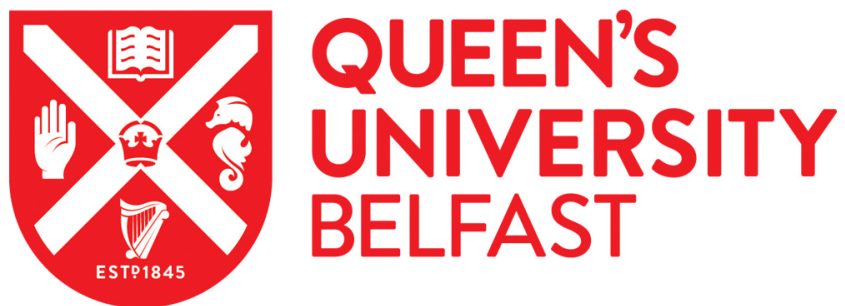

\section{Measurements of high-energy radiation generation from laser- wakefield accelerated electron beams}

Schumaker, W., Sarri, G., Vargas, M., Zhao, Z., Behm, K., Chvykov, V., Dromey, B., Hou, B., Maksimchuk, A., Nees, J., Yanovsky, V., Zepf, M., Thomas, A. G. R., \& Krushelnick, K. (2014). Measurements of high-energy radiation generation from laser-wakefield accelerated electron beams. Physics of Plasmas, 21(5), [056704]. https://doi.org/10.1063/1.4875336

Published in:

Physics of Plasmas

Document Version:

Publisher's PDF, also known as Version of record

Queen's University Belfast - Research Portal:

Link to publication record in Queen's University Belfast Research Portal

\section{Publisher rights}

(C) 2014 AIP Publishing LLC.

This article may be downloaded for personal use only. Any other use requires prior permission of the author and AIP Publishing.

The following article appeared in Schumaker, W, Sarri, G, Vargas, M, Zhao, Z, Behm, K, Chvykov, V, Dromey, B, Hou, B, Maksimchuk, A,

Nees, J, Yanovsky, V, Zepf, M, Thomas, AGR \& Krushelnick, K 2014, 'Measurements of high-energy radiation generation from laser-

wakefield accelerated electron beams' Physics of Plasmas, vol 21, no. 5, 056704 and may be found at

http://scitation.aip.org/content/aip/journal/pop/21/5/10.1063/1.4875336

\section{General rights}

Copyright for the publications made accessible via the Queen's University Belfast Research Portal is retained by the author(s) and / or other copyright owners and it is a condition of accessing these publications that users recognise and abide by the legal requirements associated with these rights.

\section{Take down policy}

The Research Portal is Queen's institutional repository that provides access to Queen's research output. Every effort has been made to ensure that content in the Research Portal does not infringe any person's rights, or applicable UK laws. If you discover content in the Research Portal that you believe breaches copyright or violates any law, please contact openaccess@qub.ac.uk. 


\section{AIP | Physisos of}

\section{Measurements of high-energy radiation generation from laser-wakefield accelerated electron beamsa)}

W. Schumaker, G. Sarri, M. Vargas, Z. Zhao, K. Behm, V. Chvykov, B. Dromey, B. Hou, A. Maksimchuk, J. Nees, V. Yanovsky, M. Zepf, A. G. R. Thomas, and K. Krushelnick

Citation: Physics of Plasmas (1994-present) 21, 056704 (2014); doi: 10.1063/1.4875336

View online: http://dx.doi.org/10.1063/1.4875336

View Table of Contents: http://scitation.aip.org/content/aip/journal/pop/21/5?ver=pdfcov

Published by the AIP Publishing

\section{Articles you may be interested in}

Improvements to laser wakefield accelerated electron beam stability, divergence, and energy spread using three-dimensional printed two-stage gas cell targets

Appl. Phys. Lett. 104, 174103 (2014); 10.1063/1.4874981

High-quality electron beam from laser wake-field acceleration in laser produced plasma plumes

Appl. Phys. Lett. 102, 231108 (2013); 10.1063/1.4810012

Generation of tunable, 100-800 MeV quasi-monoenergetic electron beams from a laser-wakefield accelerator in the blowout regimea)

Phys. Plasmas 19, 056703 (2012); 10.1063/1.4718711

Ultrashort high quality electron beam from laser wakefield accelerator using two-step plasma density profile

Rev. Sci. Instrum. 81, 033307 (2010); 10.1063/1.3360927

Subcycle high electron acceleration by crossed laser beams

Appl. Phys. Lett. 77, 1082 (2000); 10.1063/1.1289649

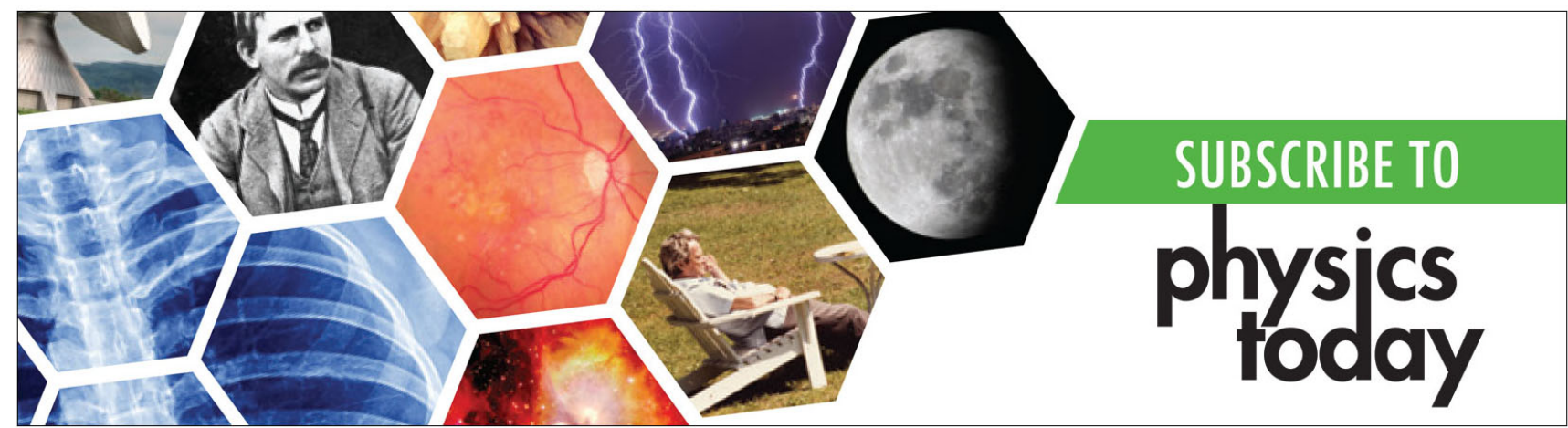




\title{
Measurements of high-energy radiation generation from laser-wakefield accelerated electron beams ${ }^{\text {a) }}$
}

\author{
W. Schumaker, ${ }^{1, b), c)}$ G. Sarri, ${ }^{2}$ M. Vargas, ${ }^{1}$ Z. Zhao, ${ }^{1}$ K. Behm, ${ }^{1}$ V. Chvykov, ${ }^{1}$ B. Dromey, ${ }^{2}$ \\ B. Hou, ${ }^{1}$ A. Maksimchuk, ${ }^{1}$ J. Nees, ${ }^{1}$ V. Yanovsky, ${ }^{1}$ M. Zepf, ${ }^{2}$ A. G. R. Thomas, ${ }^{1}$ \\ and K. Krushelnick ${ }^{1}$ \\ ${ }^{1}$ Center for Ultrafast Optical Science, University of Michigan, Ann Arbor, Michigan 48109, USA \\ ${ }^{2}$ School of Mathematics and Physics, The Queen's University of Belfast, BT7 1NN Belfast, \\ Northern Ireland, United Kingdom
}

(Received 15 January 2014; accepted 13 March 2014; published online 21 May 2014)

Using high-energy $(\sim 0.5 \mathrm{GeV})$ electron beams generated by laser wakefield acceleration (LWFA), bremsstrahlung radiation was created by interacting these beams with various solid targets. Secondary processes generate high-energy electrons, positrons, and neutrons, which can be measured shot-to-shot using magnetic spectrometers, short half-life activation, and Compton scattering. Presented here are proof-of-principle results from a high-resolution, high-energy gamma-ray spectrometer capable of single-shot operation, and high repetition rate activation diagnostics. We describe the techniques used in these measurements and their potential applications in diagnosing LWFA electron beams and measuring high-energy radiation from laserplasma interactions. @ 2014 AIP Publishing LLC. [http://dx.doi.org/10.1063/1.4875336]

\section{INTRODUCTION}

Particle accelerators are important tools for both the fundamental and applied sciences. However, conventional accelerator technology is limited in its accelerating gradient to approximately $10 \mathrm{MV} / \mathrm{m}$ by material breakdown properties, thereby requiring long acceleration lengths and, consequently, large facilities for high-energy accelerators. Alternatively, laser-driven plasma waves can sustain acceleration gradients of order $100 \mathrm{GV} / \mathrm{m}$, allowing for more compact facilities. The acceleration process of a high-intensity laser pulse driving a plasma wave to accelerate electrons is known as laser-wakefield acceleration (LWFA). ${ }^{1}$

In 2004, various groups demonstrated LWFA of monoenergetic electron beams to highly relativistic energies. ${ }^{2-4}$ In the decade following, multi-GeV energy electrons were reported using only $\mathrm{cm}$ 's of plasma. ${ }^{5-7}$ Although these beams had high-energy, mono-energetic features, their charge was relatively limited to the electron injection processes of self-trapping or colliding-pulse injection mechanisms. ${ }^{8}$ However, by adding a high-Z dopant to the plasma, the location and amount of charge can be controlled by the process of ionization injection, such that electrons are ionized at the laser's peak intensity and directly injected into the plasma wave., ${ }^{9,10}$ This allows for the separation of the injection and acceleration mechanisms in LWFA using separate stages. ${ }^{11,12}$ Under certain conditions, large amounts of charge can be injected over the course of the entire acceleration process, leading to a broad, flat energy spectra. ${ }^{12,13}$ These broad spectra, high-charge beams are well suited for bremsstrahlung generation since the process does not require

\footnotetext{
${ }^{a)}$ Paper GI2 4, Bull. Am. Phys. Soc. 58, 103 (2013).

${ }^{\mathrm{b})}$ Invited speaker.

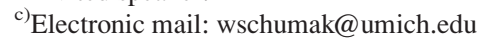

mono-energetic beams due to the inherent spectral broadening.

Since electron beams from LWFA have bunch durations on the order of the driving laser pulse $(\sim 30 \mathrm{fs}),{ }^{14,15}$ the subsequent radiation generation from these electrons is of similiar temporal duration, leading to high peak brightness ${ }^{25}$ with inherent temporal synchronization. Transverse oscillations within the plasma wave structure-termed betatron oscillations-can produce synchrotron-like X-rays with typical photon spectra centered around $10 \mathrm{keV}$ of peak brightness around $10^{22}$ photons $/ \mathrm{s} / \mathrm{mrad}^{2} / \mathrm{mm}^{2} / 1 \%$ bandwidth ${ }^{16}$ (comparable to third generation synchrotron light sources), with energies reported up to $1 \mathrm{MeV} .{ }^{17}$

To generate higher energy photons, the electron beam can be converted into bremsstrahlung by interacting with a solid target ${ }^{18}$ or through interaction with a secondary laser pulse to undergo Thomson scattering (also termed inverse Compton scattering (ICS)). ${ }^{19,20}$ With lower intensity scattering pulses (where the normalized vector potential is $a_{0}=\frac{e E_{0} \lambda_{0}}{2 \pi m_{e} c^{2}}<1$, where $E_{0}$ is the peak electric field and $\lambda_{0}$ is the wavelength of the laser pulse), the energy gain by a $180^{\circ}$ backscattered photon is $4 \gamma_{e}^{2}$, where $\gamma_{e}$ is the Lorentz factor of the electron beam. For example, a $200 \mathrm{MeV}$ electron beam $\left(\gamma_{e} \sim 400\right)$ can upshift $1.55 \mathrm{eV}$ photons ( $800 \mathrm{~nm}$ wavelength) to a $1 \mathrm{MeV}$ photons, whereas a $1 \mathrm{GeV}$ electron beam $\left(\gamma_{e}\right.$ $\sim 2000$ ) yields $25 \mathrm{MeV}$ photons, all of which are highly collimated and relatively narrow in spectral shape. ${ }^{21}$ With higher intensity $\left(a_{0} \gg 1\right)$ scattering pulses, the electrons are greatly perturbed and slowed in the scattering laser field, yielding multiple photons per electron and numerous harmonics of the upshifted fundamental ( $4 n \gamma_{e}^{2}$, where $n \sim 0.64 a_{0}$ is the brightest harmonic). ${ }^{22}$ These non-linear Thomson scattering (NLTS) effects greatly increase the yield, energy, and peak brightness of the resulting photon beam such that simulations show that a $100 \mathrm{pC}, 200 \mathrm{MeV}$ electron beam interacting with a laser pulse of $a_{0}=50$ should yield peak brightness up to 


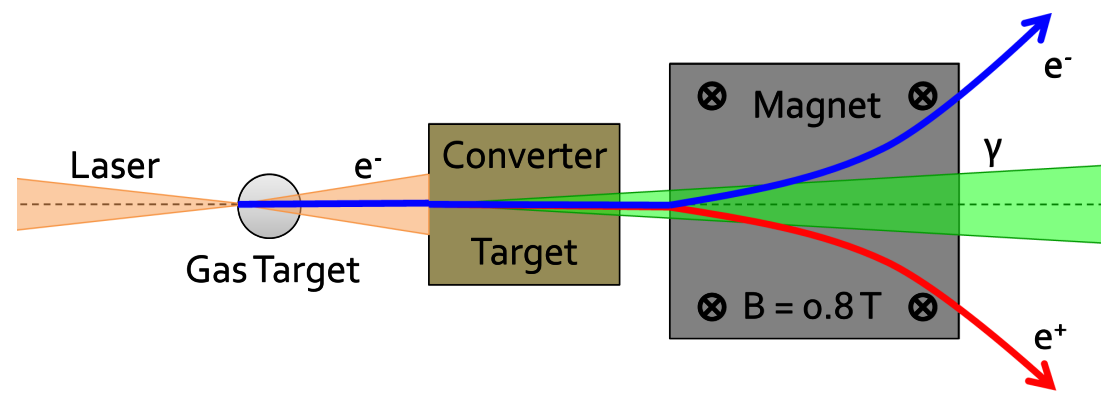

FIG. 1. Schematic of experimental geometry for bremsstrahlung radiation generation.

$10^{29}$ photons $/ \mathrm{s} / \mathrm{mrad}^{2} / \mathrm{mm}^{2} / 1 \%$ bandwidth with photon energies extending up past $30 \mathrm{MeV} .{ }^{23}$ Such peak brightness is comparable to the fourth generation light sources (X-ray free-electron lasers, XFEL), but extending to much higher energies and consisting of table-top size equipment. Moreover, such interactions may allow scientists to measure parameters where non-linear quantum electrodynamic (nQED) processes can become significant. ${ }^{24}$

In this paper, we describe proof-of-principle work which demonstrates single-shot, high-spectral resolution diagnostics of high-energy bremsstrahlung from LWFA electrons interacting with solid targets. These diagnostics are important for future NLTS experiments where high-resolution, single-shot spectral measurements are crucial for studying the underlying processes. The applications of high-energy radiation from LWFA include the production of medical isotopes (e.g., ${ }^{11} \mathrm{C},{ }^{15} \mathrm{O}$, and ${ }^{18} \mathrm{~F}$ ) ${ }^{18}$ and active interrogation/photonuclear fission of special nuclear materials (e.g., ${ }^{235} \mathrm{U},{ }^{238} \mathrm{U}$, and $\left.{ }^{239} \mathrm{Pu}\right)^{26}$ using a compact setup.

\section{EXPERIMENTAL SETUP}

For these experiments, the chirped pulse amplified, $800 \mathrm{~nm}$ Ti:sapphire-based HERCULES ${ }^{27}$ laser at the Center for Ultrafast Optical Science (CUOS) in the University of Michigan was operated between $27-100 \mathrm{TW}(0.8-3 \mathrm{~J}$ in 30 fs). The basic experimental geometry is shown in Fig. 1 . The laser was focused with an $f / 20$ off-axis paraboloid (OAP) mirror to a maximum intensity of $1.3 \times 10^{19} \mathrm{~W} / \mathrm{cm}^{2}$ $\left(a_{0}=2.5\right)$ onto either a $3 \mathrm{~mm}$ gas jet or a $1+5 \mathrm{~mm}$ staged gas cell with $\mathrm{He}$ doped with $2.5 \% \mathrm{~N}_{2}$ in the jet or first cell and pure $\mathrm{He}$ for the second cell. The resultant plasma had a peak density of $(5-10) \times 10^{18} \mathrm{~cm}^{-3}$, measured via transverse optical interferometry. In these experiments, the LWFA process generated an electron beam with a broad energy spectrum extending to $500 \mathrm{MeV}$, with up to $600 \mathrm{pC}$ of total charge, and $<2.5 \mathrm{mrad}$ divergence using the process of ionization injection ${ }^{9,10}$ (shown in Fig. 2(c)).

To characterize the electron beam before interaction with a solid converter target, a charge-calibrated magnetic spectrometer with a $0.8 \mathrm{~T}, 15 \mathrm{~cm}$ length magnet and scintillator screen (Kodak LANEX Regular) was installed directly behind the gas target in a separate shot series. Typical electron spectra are shown in Fig. 2. The magnet was also removed to measure the pointing and divergence of the electron beam with an on-axis LANEX screen, and also, in some cases, on a pixelated BGO scintillator array shielded by $50 \mathrm{~mm}$ of $\mathrm{Pb}$. For every electron diagnostic, the scintillators were cross-calibrated for charge with image plates (Fujifilm BAS-MS ${ }^{28}$ for a single shot.

Once characterized, the electron beam was propagated up to $155 \mathrm{~cm}$ before interacting with a solid converter target in a separate shot series. While passing through the converter, the electrons created bremsstrahlung photons with an energy spectrum extending up to the peak electron energy. For an ultra-relativistic electron interacting with a nucleus, experiencing a Coulomb deflection such that $E, E^{\prime} \ll M c^{2}$,

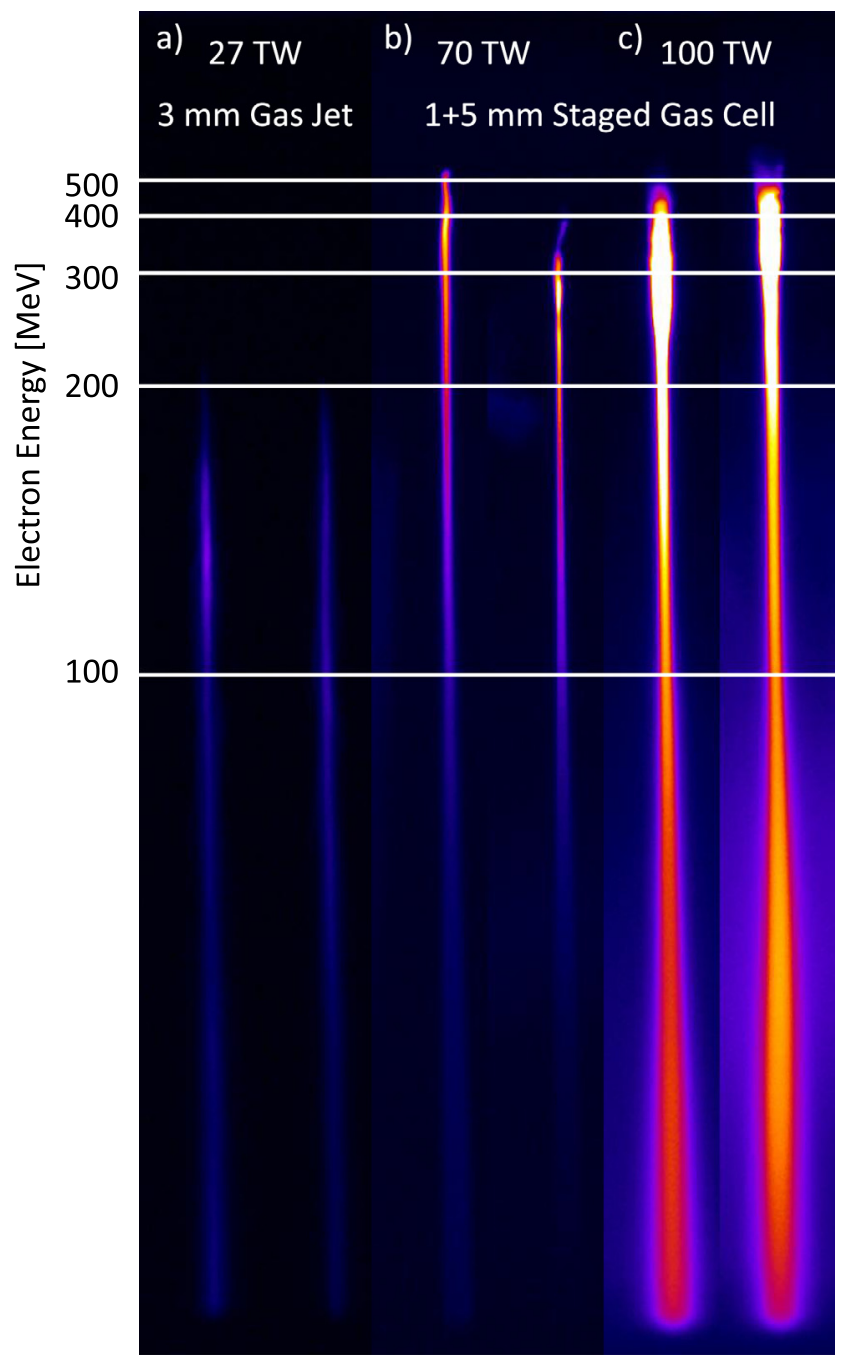

FIG. 2. Raw electron spectra used in (a) the Compton spectrometer experiment with 27 TW laser power and a $3 \mathrm{~mm}$ gas jet, (b) the autoradiography experiment with $70 \mathrm{TW}$, and (c) the activation experiments with $100 \mathrm{TW}$ and a $1+5 \mathrm{~mm}$ staged gas cell $(7 \mathrm{~mm}$ total plasma length including $1 \mathrm{~mm}$ inner separator). Note that the color scale is the same for all spectra and that the energy peaks of (c) saturated the 12-bit CCD camera. 

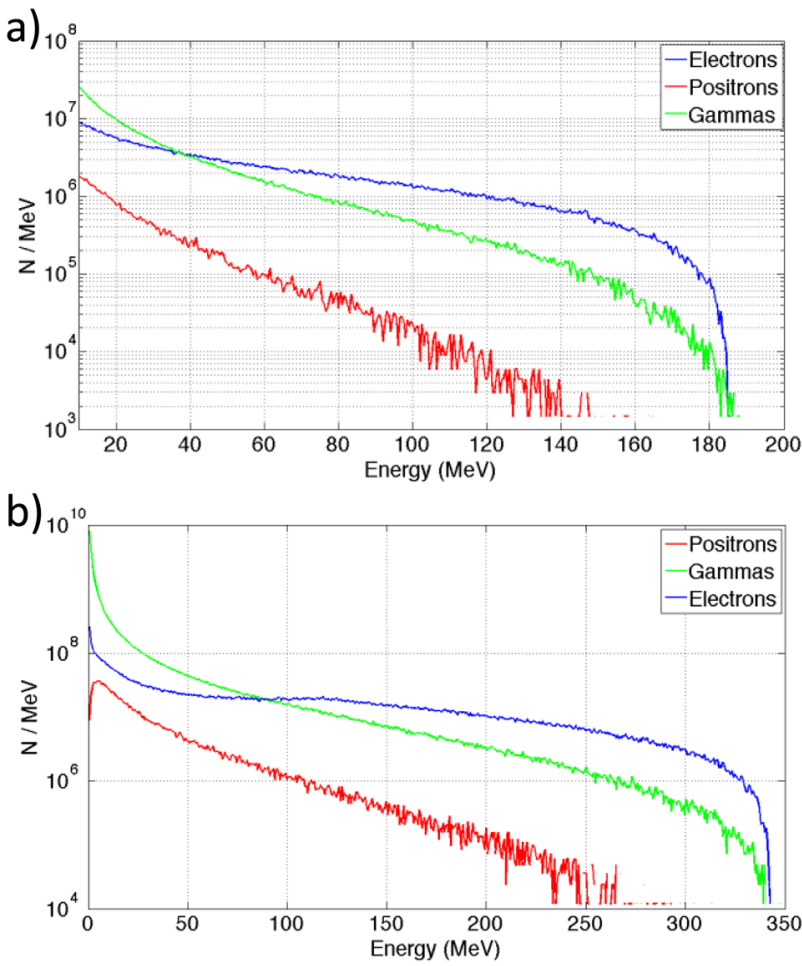

FIG. 3. Simulated bremsstrahlung spectra from a flat-top spectra electron beam with (a) $60 \mathrm{pC}$ charge up to $200 \mathrm{MeV}$ and (b) $500 \mathrm{pC}$ charge up to $350 \mathrm{MeV}$ interacting with a $2.8 \mathrm{~mm}$ Ta converter.

where $M$ is the rest mass of the nucleus and $E, E^{\prime}$ are the electron energies before and after the collision, the differential cross section for the fraction of photons radiated per unit energy interval (units of area/energy) under the Born approximation is ${ }^{29}$

$$
\begin{aligned}
\frac{d \sigma}{d(\hbar \omega)} \simeq & \frac{Z^{2} e^{6}}{12 \hbar \pi^{3} \epsilon_{0}^{3} M^{2} c^{3}}\left(1-\frac{\hbar \omega}{E}+\frac{3}{4} \frac{(\hbar \omega)^{2}}{E^{2}}\right) \\
& \times\left[\ln \left(\frac{2 E(E-\hbar \omega)}{M c^{2} \hbar \omega}\right)-\frac{1}{2}\right] \frac{1}{\hbar \omega},
\end{aligned}
$$

for $\hbar \omega / E<1$. This has a characteristic $1 / \hbar \omega$ fall off for low photon energies and a cut-off close to the initial electron energy. For low energy photons, doubly differential cross section for the fraction of photons radiated per unit energy interval per unit solid angle is

$$
\frac{d^{2} \sigma}{d(\hbar \omega) d \Omega}=\frac{3 \gamma^{2}}{2 \pi} \frac{1+\gamma^{4} \theta^{4}}{\left(1+\gamma^{2} \theta^{2}\right)^{4}} \frac{d \sigma}{d(\hbar \omega)},
$$

where $\theta$ is the emission angle. The emission is confined to a cone of angle $\theta_{c} \sim 1 / \gamma$ radians.

Depending on the converter thickness and atomic number $(Z)$, these photons can subsequently generate electronpositron pairs through a Bethe-Heitler process, ${ }^{30}$ creating copious amounts of positrons for higher $Z$ materials. The characterization of these positron beams using LWFA is published elsewhere. ${ }^{31-33}$ To corroborate the experimental results and optimize parameters, Monte-Carlo simulations using the FLUKA code were run to simulate photon and lepton creation and transport. ${ }^{34}$ Figure 3(a) shows simulated spectra from the $<200 \mathrm{MeV}, 60 \mathrm{pC}$ electron beams used for Compton scattering diagnostics (see Fig. 2(a)), whereas Figure 3(b) shows spectra using ideal LWFA electron beams of $<350 \mathrm{MeV}$ and $500 \mathrm{pC}$, similar to those used for activation experiments (see Fig. 2(c)).

For the Compton scattering diagnostic, only the photon beam is desired, so the spectrometer magnet is installed after the converter target to deflect charged particles. We also used activation diagnostics in which a solid target (either the converter itself or a secondary target) acted as a collector of radioisotopes before being read out by a slow detector.

\section{EXPERIMENTAL RESULTS}

\section{A. Compton-scattering photon spectrometer measurements}

For indirect photon spectrum measurements, we integrated 10 shots of electron beams with up to $200 \mathrm{MeV}$ energy and an average of $60 \mathrm{pC}$ of charge (Fig. 2(a)) to create photon beams as well as positrons (Fig. 3(a)). A secondary converter target of $4 \mathrm{~cm} \mathrm{LiF}$ was then used to Compton scatter these photons into secondary electrons that were spectrally separated with a magnet and detected with an image plate (see Fig. 4(a)). To collect only the on-axis secondary electrons, a $\varnothing 15 \mathrm{~mm}$ aperture, $5 \mathrm{~cm}$ length $\mathrm{Pb}$ collimator was placed before the entrance to the magnet. This ensured that only electrons in the near forward direction were measured (Fig. 5(a)). In Compton scattering, for an electron initially at rest the final energy of the electron, $E$, depends on the scattered angle and is given by

$$
E=\left(1-\left[\frac{\hbar \omega}{m_{e} c^{2}}(1-\cos \theta)+1\right]^{-1}\right) \hbar \omega,
$$

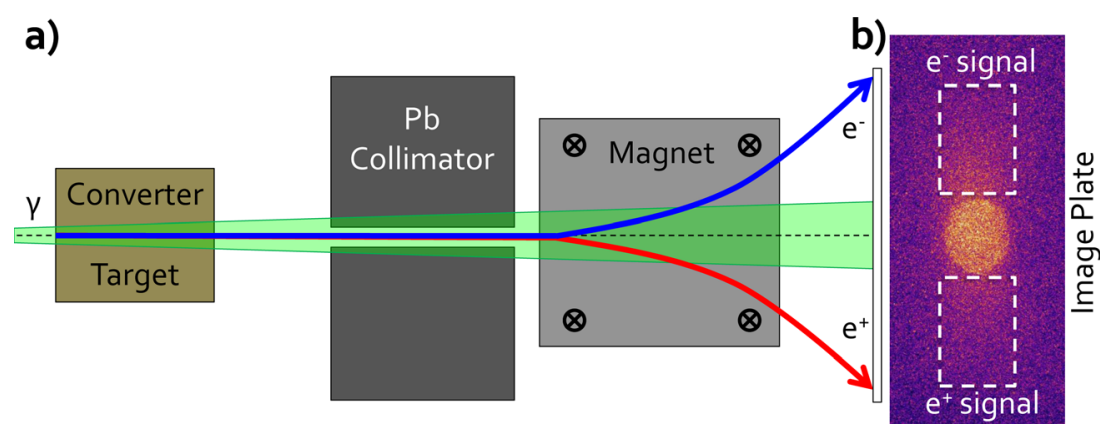

FIG. 4. (a) Compton-scattering spectrometer geometry. Note that the $\mathrm{Pb}$ collimator blocks the off-axis charged particles but can cause contaminant signal as a converter. (b) Raw image plate signal with outlined regions-ofinterest. The signal in the center is from the on-axis photon beam. 

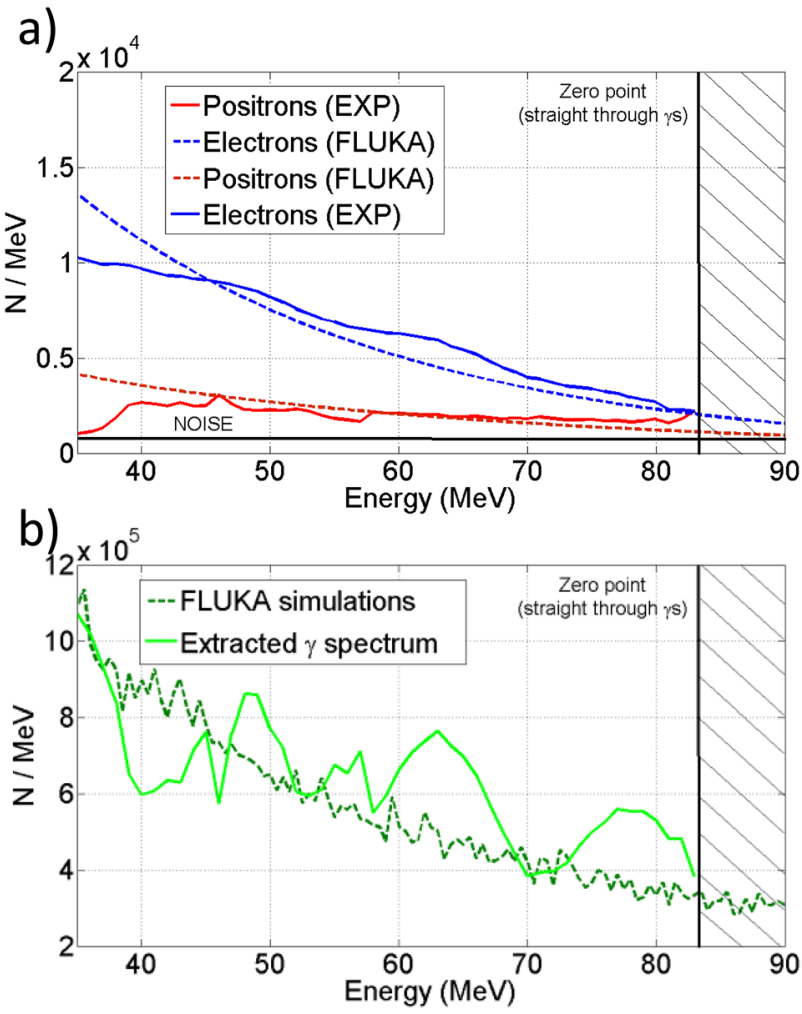

FIG. 5. (a) Measured (solid) and simulated (dashed) spectra for secondary electrons/positrons from Compton scattering. (b) Extracted (light green) and simulated (dark green) photon spectra based on electron/positron energies. Simulated input photon spectra were used from Fig. 3(a).

where $\theta$ is the angle between the momentum of the incident photon (with energy $\hbar \omega$ ) and the scattered photon. Therefore, for $\hbar \omega \gg m_{e} c^{2}$ and backward scattering, $\theta=\pi$, the forward scattered electron will have energy

$$
E=\hbar \omega-\frac{m_{e} c^{2}}{2}
$$

i.e., very close to the original photon energy. The photon spectrum can be determined from the electron spectrum after taking this shift into account. However, in addition to this downshift in energy, scattering within the solid material will also affect the measured electron energy spectrum (Fig. 5(a)). Monte Carlo modeling can be used to determine an electron spectrum for incident photons of a certain energy (Fig. 6). The photon spectrum can subsequently be obtained from the electron spectrum by deconvolution (Fig. 5(b)). The positron signal indicates the amount of pair production occurring in the converter and must be subtracted from the total electron signal to isolate the Compton scattered electron signal (Fig. 5(a)).

To minimize pair production background and electron scattering losses in the converter, a low- $Z$ material is preferred as pair production and stopping power scale approximately with $Z^{2}$. Hence, relatively low- $Z$ and dense $\mathrm{LiF}$ was chosen for an initial converter material. Compared to elemental hydrogen, LiF does broaden the spectrum of exiting electrons but allows for higher yield. This enables LiF to measure peak photon energy and overall flux but with lower spectral resolution. This is shown in FLUKA simulations where $30 \mathrm{MeV}$

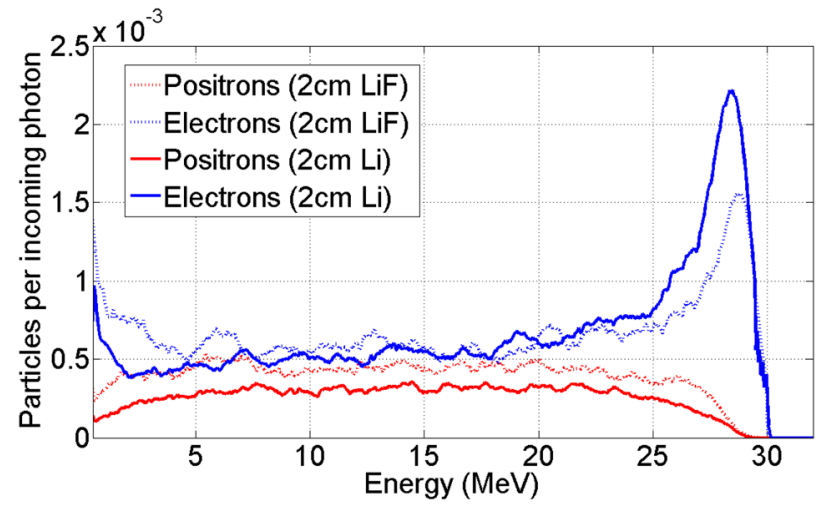

FIG. 6. Simulated spectra of secondary electrons (red) and positrons (green) emitted on-axis from $30 \mathrm{MeV}$ mono-energetic photons interacting with $2 \mathrm{~cm}$ lithium (solid) and $2 \mathrm{~cm} \mathrm{LiF} \mathrm{(dashed)} \mathrm{converter} \mathrm{target.}$

mono-energetic photons interacted with $2 \mathrm{~cm} \mathrm{Li}$ and $\mathrm{LiF}$ converters and the exiting electron and positron energies were measured (see Fig. 6). One other limitation to the spectrometer is the aperture material and geometry. Ideally, the aperture would allow only the on-axis electrons into the spectrometer, but since the photon beam is generally larger than the aperture, there will be particle conversion within the aperture itself. By maximizing the aperture material $Z$, the scattering and stopping power are maximized so that the background contamination is uniform, but weak in energy. Therefore, the aperture size affects the spectral resolution in two ways: smaller apertures restrict the acceptance angle and narrow the spectral bandwidth at the cost of creating more background from interaction with the aperture material and cutting incident signal. Lastly, the aperture size also dictates the zero-point (unresolved area) on the detector based on projection (seen in Fig. 4). As a result, a moderate size aperture was chosen to increase flux at the expense of spectral resolution.

\section{B. $(\gamma, \mathrm{n})$ Activation measurements}

When a energetic photon has enough energy to overcome the binding energy of an neutron in the nucleus (7-20 MeV per neutron), it can dislodge a neutron with a small, but significant, probability. When a neutron is ejected, the remaining proton-rich nucleus is typically radioactive and undergoes $\beta^{+}$decay thereby emitting a positron. This positron quickly annihilates with a nearby electron to give off two $511 \mathrm{keV}$ photons in opposing directions which can be easily measured and distinguished from background with a pair of NaI scintillators in coincidence (Fig. 7). The normalized activity curves for different materials irradiated by $10^{6}$ photons of $20 \mathrm{MeV}$ energy are shown in Fig. 8(a). Utilizing materials with few second half-lives allows the coincident $511 \mathrm{keV}$ signal to be clearly distinguished from the prompt radiation due to the electron beam and effectively collected (over numerous half-lives) before the next shot (in minute intervals). For these measurements, silicon was chosen as an activation target due to the short $(4.15 \mathrm{~s})$ half-life of its primary $(\gamma, \mathrm{n})$ activation product $\left({ }^{27} \mathrm{Si}\right)$ and its high-energy $(\sim 16 \mathrm{MeV})$ reaction threshold.

The detector geometry and reaction process are depicted in Fig. 7. The NaI scintillators were both $\varnothing 75 \mathrm{~mm} \times 75 \mathrm{~mm}$ 


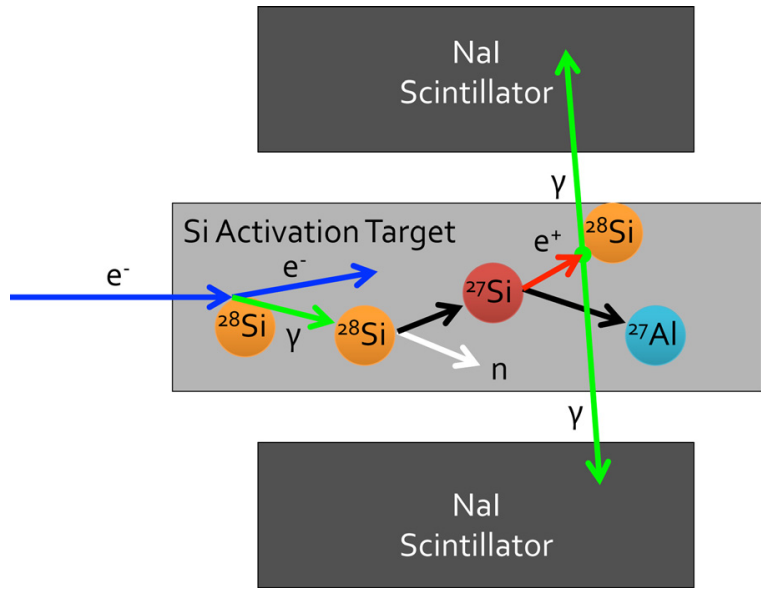

FIG. 7. Nuclear reaction diagram for ${ }^{28} \mathrm{Si}$ activation. Bremsstrahlung photons dislodge neutrons from ${ }^{28} \mathrm{Si}$ to give ${ }^{27} \mathrm{Si}$ which undergoes $\beta^{+}$decay (half-life of $4.15 \mathrm{~s}$ ) and emits a $e^{+}$which quickly annihilates with a nearby $e^{-}$to give two $511 \mathrm{keV}$ photons.

in size, separated by $10 \mathrm{~cm}(5 \mathrm{~cm}$ on either side of the axis), and shielded with lead and graphite. High-purity (>99.999\%), monocrystalline silicon of $10 \mathrm{~mm} \times 10 \mathrm{~mm}$ $\times 100 \mathrm{~mm}$ size was placed on axis with the electron beam $155 \mathrm{~cm}$ away from the gas cell. Before entering the activation sample, the broad-energy electron beam passed through a $600 \mu \mathrm{m}$ thick beryllium window $10 \mathrm{~cm}$ in front of the sample. The coincidence counting electronics were temporally gated until $100 \mathrm{~ms}$ after the shot to avoid prompt radiation from the electron beam, were energy gated for $450 \pm 100 \mathrm{keV}$ using single-channel analyzers (SCA) to remove spurious coincidences, and digitally read-out for real-time analysis. Over the course of 20 shots of electrons with up to $500 \mathrm{MeV}$ energy and an average of $600 \mathrm{pC}$ of charge (Fig. 2(c)), the measured activation varied with the pointing and charge of the beam as shown in Fig. 8(b).
Overall, the measured activity for an average shot had a halflife of $4.06 \pm 0.67 \mathrm{~s}$ and an initial activation of $648 \pm 320$ decays as estimated by fitting individual. The low number of measured decays was likely due to the scatter of the beam before entering the sample and the inefficient bremsstrahlung conversion within the relatively low- $Z$ sample. However, given the $0.12 \%$ probability of a photon inducing a reaction in the rod, the estimated $>15 \mathrm{MeV}$ photon flux is on the order of $5 \times 10^{5}$ photons $/ \mathrm{cm}^{2} /$ shot.

Alternatively, fused silica $\left(\mathrm{SiO}_{2}\right)$ and copper rods of $\emptyset 12.7 \mathrm{~mm} \times 100 \mathrm{~mm}$ size were used to demonstrate different aspects of activation. An activity trace of electron beams entering fused silica is shown in Fig. 8(c). Activation of oxygen (122 s half-life) in the fused silica sample establishes a low-level background between the short-lived silicon peaks. To investigate longer half-life materials, 25 shots were taken on a $\mathrm{Cu}$ sample $\left({ }^{62} \mathrm{Cu}\right.$ half-life is $\left.9.67 \mathrm{~min}\right)$. The $\mathrm{Cu}$ activity clearly follows the expected exponential decay as shown in Fig. 8(d). Assuming each shot contributed roughly the same signal and taking into account the time between shots, a simple rate equation can model the build-up of activity until shots cease. By fitting the decay curve of $\mathrm{Cu}$ to yield the initial activity after shots and in conjuction with this simple model, a estimate of the activations for a single-shot can be estimated as 4200 decays/shot, corresponding to $1.4 \times 10^{5}$ photons/shot above $10 \mathrm{MeV}$ given the $3 \%$ probability of interaction.

\section{C. $(\gamma, \mathrm{n})$ Autoradiography measurements}

Besides measuring the exponential decay of the activation signal, the spatial distribution of activation can be measured by placing a spatially sensitive, integrating detector (such as imaging plate, film, CCD, etc.) on the sample after the shot in a process known as autoradiography. In our

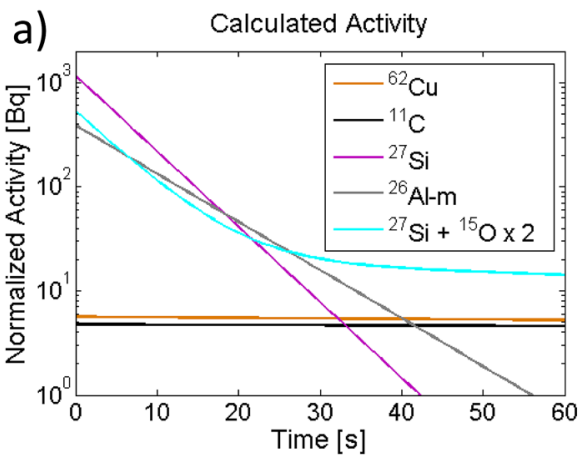

c)

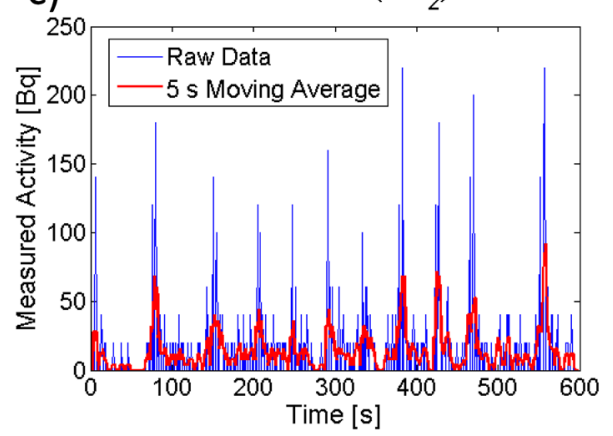

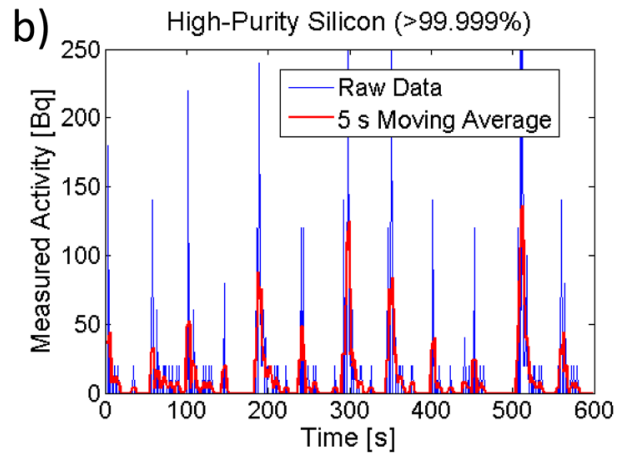

d)

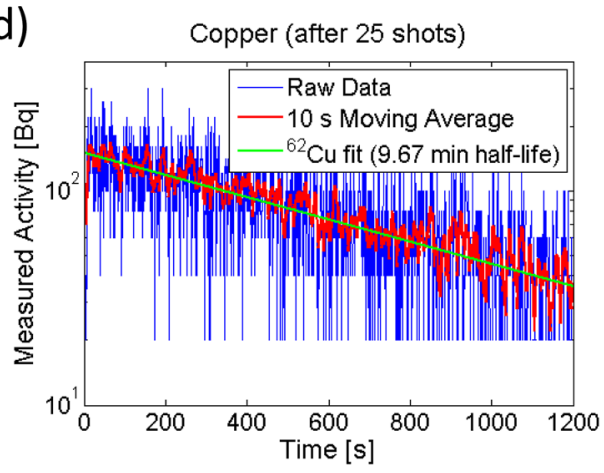

FIG. 8. (a) Calculated activities for various materials irradiated by $10^{6}$ photons of $20 \mathrm{MeV}$ energy normalized according to abundance and density. (b) and (c) Experimental (blue) and averaged (red) activity traces from multiple shots on (b) high-purity $\mathrm{Si}$ and (c) fused silica $\left(\mathrm{SiO}_{2}\right)$. (d) Experimental activity (blue), averaged (red), and expected (green) traces of 25 shots accumulation on $\mathrm{Cu}$. 
a)
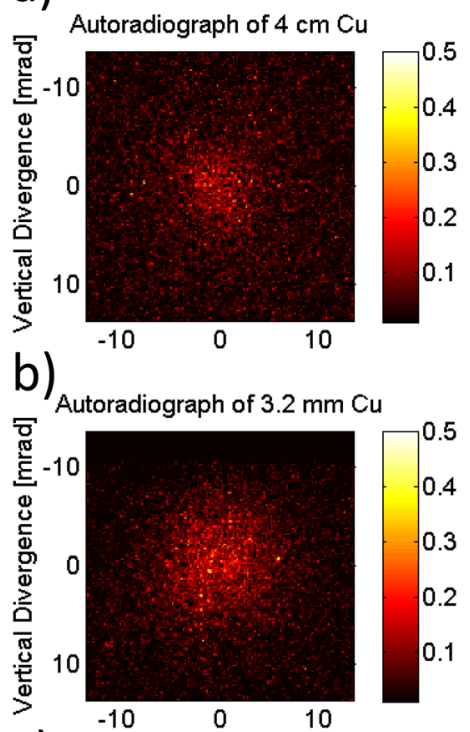

c)

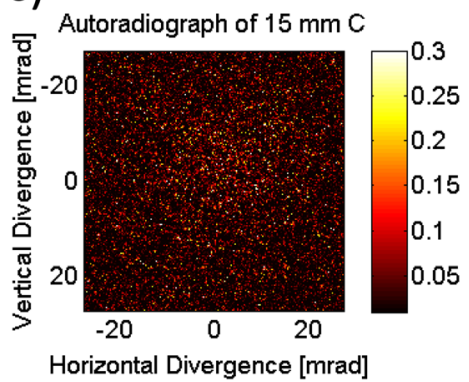

d)

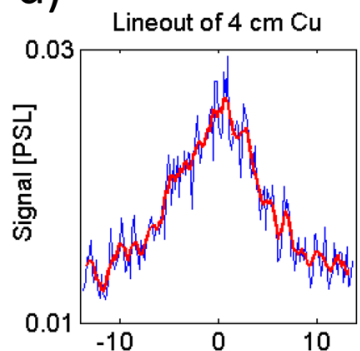

e)

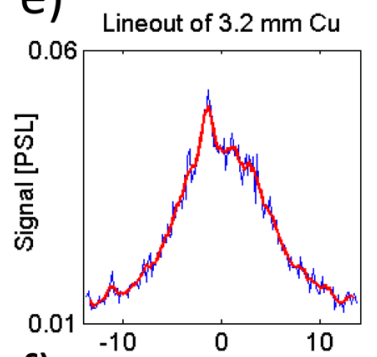

f)

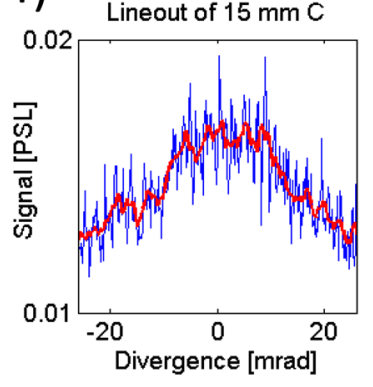

FIG. 9. Autoradiographs of 20 electron beams passing through (a) $4 \mathrm{~cm}$ of $\mathrm{Cu}$, (b) $3.2 \mathrm{~mm}$ of $\mathrm{Cu}$, and (c) $15 \mathrm{~mm}$ of $\mathrm{C}$ in units of photostimulated luminescence (PSL). (d)-(f) Horizontal line-outs taken across each respective image. Note that (a) and (b) are on the same scale.

configuration, one of the two escaping $511 \mathrm{keV}$ photon was recorded spatially on the image plate and over the course of numerous decays produced an image of the activation spatial distribution. Since the image plates must be installed after the shot to prevent background, this means the sample must be able to hold the activity for $\sim$ minutes in order to be transferred and subsequently read-out. As such, copper and carbon targets were chosen for their moderate half-lives of 9.67 and 20.33 min (see Fig. 8(a)), respectively, and their different $(\gamma, \mathrm{n})$ activation energy thresholds $(10$ and $20 \mathrm{MeV}$, respectively). The separate energy bins enables a notched gamma-ray spectrometer to spatially measure photons above threshold energy with essentially zero background from the low-energy portion of the spectrum. For photon spectra extending to significantly higher energies (as in our experiment), the ability to measure differences across these spectral bins becomes marginal. However, for photon spectra in this range, the ability to measure these two spectral components accurately is useful for characterizing exponential tails of low energy spectra ${ }^{35,36}$ and the peak photon energy of broad spectra beams.

For the experiment, we placed various samples of copper and graphite on-axis with the $500 \mathrm{MeV}$ peak, $180 \mathrm{pC}$ average charge electron beam (Fig. 2(b)) in place of the silicon sample as described in Sec. III B. The stack consisted of

$4 \mathrm{~cm} \mathrm{Cu}, 3.2 \mathrm{~mm} \mathrm{Cu}$, and $15 \mathrm{~mm} \mathrm{C}$ in order of increasing distance from the gas target where the $4 \mathrm{~cm} \mathrm{Cu}$ acted as the primary converter. The plates were integrated for 20 shots and were read out by separate image plates for $1 \mathrm{~h}$ (collecting 98\% and $88 \%$ of the signal for $\mathrm{Cu}$ and $\mathrm{C}$, respectively). The spatial profiles of the electron beam impacting the $4 \mathrm{~cm} \mathrm{Cu}$, $3.2 \mathrm{~mm} \mathrm{Cu}$, and $15 \mathrm{~mm} \mathrm{C}$ are shown in Figs. 9(a)-9(c), respectively. Taking a lineout across each signal (Figs. 9(d)-9(f)), the divergence of the beam on $4 \mathrm{~cm}$ and $3.2 \mathrm{~mm}$ $\mathrm{Cu}$ was measured to be roughly $10 \mathrm{mrad}$ full-width-at-halfmaximum (FWHM). Since $511 \mathrm{keV}$ photons are the primary contributors to the signal, the image plate signal can also be integrated to estimate the total photon flux. For the $3.2 \mathrm{~mm}$ $\mathrm{Cu}$, total activations were $2 \times 10^{4}$ activations/shot, implying a $10 \mathrm{MeV}$ flux of $2 \times 10^{7}$ photons $/ \mathrm{cm}^{2}$ given approximately $0.1 \%$ probability of capture in the $1 \mathrm{~cm}^{2}$ area and $3.2 \mathrm{~mm}$ thickness.

One important consideration is the thickness of the sample. Thicker samples undergo more activations, but the detector collects scattered photons and secondary particles from deep in the sample and photons travelling greater distances before exiting the sample, thereby increasing the background. This effect was observed between the $4 \mathrm{~cm}$ and $3.2 \mathrm{~mm}$ samples of $\mathrm{Cu}$.

\section{CONCLUSIONS}

In conclusion, electron beams from LWFA can be used for a variety of high-energy, high-brightness radiation generation mechanisms. In this paper, we have demonstrated proof-of-principle, single-shot diagnostics which can be used at high repetition rate for measuring this high-energy radiation. The first single-shot, high-resolution gamma-ray spectrum from LWFA bremsstrahlung was measured using a Compton-scattering based electron spectrometer. Moreover, the first measurements of short-lived (few second) radioisotopes using a LWFA source enable a shot-to-shot energy monitoring diagnostic of the secondary radiation.

\section{ACKNOWLEDGMENTS}

This research was supported by DOE/NSF-PHY 0810979, NSF CAREER 1054164, DARPA AXiS N6600111-1-4208, SF/DNDO F021166, and the Leverhulme Trust ECF-2011-383.

${ }^{1}$ T. Tajima and J. M. Dawson, Phys. Rev. Lett. 43, 267 (1979).

${ }^{2}$ J. Faure, Y. Glinec, A. Pukhov, S. Kiselev, S. Gordienko, E. Lefebvre, J.-P. Rousseau, F. Burgy, and V. Malka, Nature 431, 541 (2004).

${ }^{3}$ C. G. R. Geddes, C. Toth, J. van Tilborg, E. Esarey, C. B. Schroeder, D. Bruhwiler, C. Nieter, J. Cary, and W. P. Leemans, Nature 431, 538 (2004). ${ }^{4}$ S. P. D. Mangles, C. D. Murphy, Z. Najmudin, A. G. R. Thomas, J. L. Collier, A. E. Dangor, E. J. Divall, P. S. Foster, J. G. Gallacher, C. J. Hooker et al., Nature 431, 535 (2004).

${ }^{5}$ W. P. Leemans, B. Nagler, A. J. Gonsalves, Cs. Toth, K. Nakamura, C. G. R. Geddes, E. Esarey, C. B. Schroeder, and S. M. Hooker, Nat. Phys. 2, 696 (2006)

${ }^{6}$ S. Kneip, S. R. Nagel, S. F. Martins, S. P. D. Mangles, C. Bellei, O. Chekhlov, R. J. Clarke, N. Delerue, E. J. Divall, G. Doucas, K. Ertel, F. Fiuza, R. Fonseca, P. Foster, S. J. Hawkes, C. J. Hooker, K. Krushelnick, W. B. Mori, C. A. J. Palmer, K. Ta Phuoc, P. P. Rajeev, J. Schreiber, M. J. 
V. Streeter, D. Urner, J. Vieira, L. O. Silva, and Z. Najmudin, Phys. Rev. Lett. 103, 035002 (2009).

${ }^{7}$ X. Wang, R. Zgadzaj, N. Fazel, Z. Li, S. A. Yi, X. Zhang, W. Henderson, Y.-Y. Chang, R. Korzekwa, H.-E. Tsai, C.-H. Pai, H. Quevedo, G. Dyer, E. Gaul, M. Martinez, A. C. Bernstein, T. Borger, M. Spinks, M. Donovan, V. Khudik, G. Shvets, T. Ditmire, and M. C. Downer, Nat. Commun. 4, 1988 (2013).

${ }^{8}$ J. Faure, C. Rechatin, A. Norlin, A. Lifschitz, Y. Glinec, and V. Malka, Nature 444, 737 (2006).

${ }^{9}$ C. McGuffey, A. G. R. Thomas, W. Schumaker, T. Matsuoka, V. Chvykov, F. J. Dollar, G. Kalintchenko, V. Yanovsky, A. Maksimchuk, K. Krushelnick et al., Phys. Rev. Lett. 104, 025004 (2010).

${ }^{10}$ A. Pak, K. A. Marsh, S. F. Martins, W. Lu, W. B. Mori, and C. Joshi, Phys. Rev. Lett. 104, 025003 (2010).

${ }^{11}$ B. B. Pollock, C. E. Clayton, J. E. Ralph, F. Albert, A. Davidson, L. Divol, C. Filip, S. H. Glenzer, K. Herpoldt, W. Lu et al. Phys. Rev. Lett. 107, 045001 (2011).

${ }^{12}$ M. Vargas, W. Schumaker, V. Chvykov, A. Maksimchuk, V. Yanovsky, K. Krushelnick, and A. G. R. Thomas, Appl. Phys. Lett. (submitted).

${ }^{13}$ C. E. Clayton, J. E. Ralph, F. Albert, R. A. Fonseca, S. H. Glenzer, C. Joshi, W. Lu, K. A. Marsh, S. F. Martins, W. B. Mori et al., Phys. Rev. Lett. 105, 105003 (2010).

${ }^{14}$ S. P. D. Mangles et al., Phys. Rev. Lett. 96, 215001 (2006).

${ }^{15}$ O. Lundh, J. Lim, C. Rechatin, L. Ammoura, A. Ben-Ismail, X. Davoine, G. Gallot, J.-P. Goddet, E. Lefebvre, V. Malka et al., Nat. Phys. 7, 219 (2011).

${ }^{16}$ S. Kneip, C. McGuffey, J. L. Martins, S. F. Martins, C. Bellei, V. Chvykov, F. Dollar, R. Fonseca, C. Huntington, G. Kalintchenko et al., Nat. Phys. 6, 980 (2010).

${ }^{17}$ S. Cipiccia, M. R. Islam, B. Ersfeld, R. P. Shanks, E. Brunetti, G. Vieux, X. Yang, R. C. Issac, S. M. Wiggins, G. H. Welsh et al., Nat. Phys. 7, 867 (2011)

${ }^{18}$ W. P. Leemans, D. Rodgers, P. E. Catravas, C. G. R. Geddes, G. Fubiani, E. Esarey, B. A. Shadwick, R. Donahue, and A. Smith, Phys. Plasmas 8, 2510 (2001).

${ }^{19}$ K. Ta Phuoc, S. Corde, C. Thaury, V. Malka, A. Tafzi, J.-P. Goddet, R. C. Shah, S. Sebban, and A. Rousse, Nat. Photonics 6, 308 (2012).

${ }^{20}$ S. Chen, N. D. Powers, I. Ghebregziabher, C. M. Maharjan, C. Liu, G. Golovin, S. Banerjee, J. Zhang, N. Cunningham, A. Moorti et al., Phys. Rev. Lett. 110, 155003 (2013).

${ }^{21}$ F. V. Hartemann, F. Albert, C. W. Siders, and C. P. J. Barty, Phys. Rev. Lett. 105, 130801 (2010).
${ }^{22}$ Y.-Y. Lau, F. He, D. P. Umstadter, and R. Kowalczyk, Phys. Plasmas 10, 2155 (2003).

${ }^{23}$ A. G. R. Thomas, C. P. Ridgers, S. S. Bulanov, B. J. Griffin, and S. P. D. Mangles, Phys. Rev. X 2, 041004 (2012).

${ }^{24}$ A. Di Piazza, C. Müller, K. Z. Hatsagortsyan, and C. H. Keitel, Rev. Mod. Phys. 84, 1177 (2012).

${ }^{25}$ S. Corde, K. Ta Phuoc, G. Lambert, R. Fitour, V. Malka, A. Rousse, A. Beck, and E. Lefebvre, Rev. Mod. Phys. 85, 1 (2013).

${ }^{26}$ S. A. Reed, V. Chvykov, G. Kalintchenko, T. Matsuoka, P. Rousseau, V. Yanovsky, C. R. Vane, J. R. Beene, D. Stracener, D. R. Schultz, and A. Maksimchuk, Appl. Phys. Lett. 89, 231107 (2006).

${ }^{27}$ V. Yanovsky, V. Chvykov, G. Kalinchenko, P. Rousseau, T. Planchon, T. Matsuoka, A. Maksimchuk, J. Nees, G. Cheriaux, G. Mourou et al., Opt. Express 16, 2109 (2008).

${ }^{28}$ K. A. Tanaka, T. Yabuuchi, T. Sato, R. Kodama, Y. Kitagawa, T. Takahashi, T. Ikeda, Y. Honda, and S. Okuda, Rev. Sci. Instrum. 76, 013507 (2005).

${ }^{29}$ J. D. Jackson, Classical Electrodynamics, 3rd ed. (John Wiley and Sons Inc., New York, 1999).

${ }^{30}$ H. Bethe and W. Heitler, Proc. R. Soc. London, Ser. A 146, 83 (1934).

${ }^{31}$ G. Sarri, W. Schumaker, A. Di Piazza, M. Vargas, B. Dromey, M. E. Dieckmann, V. Chvykov, A. Maksimchuk, V. Yanovsky, Z. He et al., Phys. Rev. Lett. 110, 255002 (2013).

${ }^{32}$ G. Sarri, W. Schumaker, A. Di Piazza, K. Poder, J. M. Cole, M. Vargas, D. Doria, S. Kushel, B. Dromey, G. Grittani, L. Gizzi, M. E. Dieckmann, A. Green, V. Chvykov, A. Maksimchuk, V. Yanovsky, Z. He, B. Hou, J. A. Nees, S. Kar, Z. Najmudin, A. G. R. Thomas, C. H. Keitel, K. Krushelnick, and M. Zepf, Plasma Phys. Controlled Fusion 55, 124017 (2013).

${ }^{33}$ G. Sarri, K. Poder, J. M. Cole, W. Schumaker, A. Di Piazza, B. Reville, D. Doria, M. Vargas, S. Kar, S. Kushel, A. Green, B. Dromey, G. Grittani, L. Gizzi, A. G. R. Thomas, S. P. D. Mangles, Z. Najmudin, C. H. Keitel, K. Krushelnick, and M. Zepf, Phys. Rev. Lett. (submitted); preprint arXiv: 1312.2011.

${ }^{34}$ G. Battistoni, F. Cerutti, A. Fasso, A. Ferrari, S. Muraro, J. Ranft, S. Roesler, and P. R. Sala, AIP Conf. Proc. 896, 31 (2007).

${ }^{35}$ C. Courtois, A. Compant La Fontaine, O. Landoas, G. Lidove, V. Méot, P. Morel, R. Nuter, E. Lefebvre, A. Boscheron, J. Grenier et al., Phys. Plasmas 16, 013105 (2009).

${ }^{36}$ K. W. D. Ledingham, I. Spencer, T. McCanny, R. P. Singhal, M. I. K. Santala, E. Clark, I. Watts, F. N. Beg, M. Zepf, K. Krushelnick et al., Phys. Rev. Lett. 84, 899 (2000). 